\title{
AUTOMATIC COMPUTATION OF CDR USING FUZZY CLUSTERING TECHNIQUES
}

\author{
Thresiamma Devasia ${ }^{1}$, Poulose $\mathrm{Jacob}^{2}$ and Tessamma Thomas ${ }^{3}$ \\ ${ }^{1}$ Department of Computer Science, Assumption College Changanacherry, \\ Kerala, India \\ cherukusumamegmail.com \\ ${ }^{2}$ Department of Computer Science, \\ Cochin University of Science and Technology, Kerala, India \\ kpj0101@gmail.com \\ ${ }^{3}$ Department of Electronics, \\ Cochin University of Science and Technology, Kerala, India \\ tessecusat.ac.in
}

\begin{abstract}
Eye disease identification techniques are highly important in the field of ophthalmology. A vertical Cup-to-Disc Ratio which is the ratio of the vertical diameter of the optic cup to that of the optic disc, of the fundus eye image is one of the important signs of glaucoma. This paper presents an automated method for the extraction of optic disc and optic cup using Fuzzy C Means clustering technique. The validity of this new method has been tested on 454 colour fundus images from three different publicly available databases DRION, DIARATDBO and DIARETDB1 and, images from an ophthalmologist. The average success rate of optic disc and optic cup segmentation is 94.26percentage. The scatter plot depicts high positive correlation between clinical CDR and the CDR obtained using the new method. The result of the system seems to be promising and useful for clinical work.
\end{abstract}

\section{KEYWORDS}

Fundus image, optic disc, optic cup, Cup-to-Disc Ratio

\section{INTRODUCTION}

The fundus images are used for diagnosis by trained clinicians to check for any abnormalities or any change in the retina. Important anatomical structures captured in a fundus image are blood vessels, optic cup (OC), optic disc (OD) and macula for a normal retina. An image of a diseased retina may also contain many visible symptoms of the eye-disease. In a healthy retinal image the OD usually appears as a circular shaped bright yellowish object which is partly covered with vessels. The optic cup is the cupping of the optic nerve and that means the size of the depression in the middle of the nerve when viewed from the front of the eye. When there is damage to the optic nerve, the cupping increases. Changes in the OD and OC can indicate the presence, current state and progression of glaucoma [1][2]. An efficient segmentation of OD and OC is essential to diagnose various stages of certain diseases like glaucoma. The automatic computation of Cup-toDisc Ratio (CDR) helps the ophthalmologist to do the screening and detection of glaucoma easily. In this paper, the vertical CDR is calculated by using fundus photograph where vertical CDR, is an important indicator of glaucoma [3]. Since the colour fundus images provide early signs of certain diseases such as diabetes, glaucoma etc., colour fundus images are used to track the eye

Dhinaharan Nagamalai et al. (Eds) : CSTY, SIGI, AI, MaVaS - 2015

pp. 27-40, 2015. (C) CS \& IT-CSCP 2015

DOI : $10.5121 /$ csit.2015.50904 
diseases by the ophthalmologists. Figure1 shows the important features of a retinal colour fundus image.

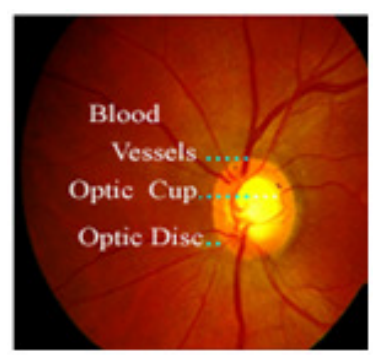

Figure 1. Colour Fundus Image

This paper is organized as follows:

Section II presents a brief survey of existing literature. Section III describes the materials used for the present work. A new algorithm to efficiently extract optic disc and optic cup in ocular fundus images is given in Section IV. The results are presented in Section V, and Conclusions are given in the final Section VI.

\section{LITERATURE SURVEY}

The Active Shape Model (ASM) based optical disk detection is implemented by Huiqi et al.[4]. The initialization of the parameters for this model is based on Principal Component Analysis technique. The faster convergence rate and the robustness of the technique are proved by experimental results. Huajun Ying et al. [5] designed a fractal-based automatic localization and segmentation of optic disc in retinal images. K. Shekar [6] developed a method for OD segmentation using Hussain, A.R. et al. [7] proposed a method for optic nerve head segmentation using genetic active contours. Zhuo Zhang et al. [8] designed a convex hull based neuro-retinal optic cup ellipse optimization technique. Wong, D.W.K. et al. [9] developed SVM-based model optic cup detection for glaucoma detection using the cup to disc ratio in retinal fundus images. Joshi G.D. et al. [10] developed vessel bend-based cup segmentation in retinal images. Shijian Lu et al. [11] proposed a background elimination method for the automatic detection of OD. Morphological operations were used for locating the optic disc in retinal images by Angel Suero et al. [12].

In this paper, a new algorithm based on Fuzzy C-Means Clustering (FCM) technique combined with thresholding, is used for OD and OC extraction. This new method, firstly, extracts the OD and OC of the colour fundus image and computes the vertical CDR automatically. This is an efficient method for the automatic screening of colour fundus image for CDR computation.

\section{MATERIALS AND METHODS}

The fundus images used in these experiments are taken from publicly available databases DRION, DIARATDB0 and DIARETDB1 and, images from Giridhar Eye Institute, Kochi, Kerala. The CDRs obtained from an ophthalmologist is used as ground truth for the evaluation.

\section{DEVELOPED ALGORITHM}

The new approach is composed of four steps. The channels of the colour retinal are separated. The blood vessels are removed, applying the contrast adjustment to enhance the low contrast 
image image. The Fuzzy C Means combined with thresholding is applied on the red channel of the input image for the extraction of the OD and the same technique is applied on the green channel of the input image for the extraction of OC. The CDR is computed using the ratio of vertical diameter of OC and OD.

\subsection{Preprocessing}

The preprocessing step excludes variations due to image acquisition, such as inhomogeneous illumination. In preprocessing, techniques such as morphological operations and contrast enhancement are applied on the input image [13]. The following sections include different preprocessing operations used in this paper.

\subsubsection{Preprocessing steps for Optic Disc Extraction}

\subsubsection{Selection of Red Channel}

From the previous studies it is shown that even though the green component of an RGB retinography is the one with highest contrast, the OD is often present in the red field as a welldefined white shape, brighter than the surrounding area [14]. Therefore the red channel of the RGB colour images is used for the extraction of OD regions in the retinal fundus images.

\subsubsection{Removal of Blood Vessels}

Since blood vessels within the OD are strong distracters, they should be erased from the image beforehand. In this method a morphological closing operation is performed on the red channel. The dilation operation first removes the blood vessels and then the erosion operation approximately restores the boundaries to their former position.

$$
\text { Closing } \quad C(A, B)=A \bullet B=E(D(A,-B),-B)
$$

where $\mathrm{A}$ is the red channel of the input image and $\mathrm{B}$ is a $10 \mathrm{x} 10$ symmetrical disc structuring element, to remove the blood vessels[15]. $\mathrm{C}$ is the resultant vessel free, smoothed output image.

\subsubsection{Preprocessing steps for Optic Cup Extraction}

\subsubsection{Selection of Green Channel}

The green channel has low contrast variation which gives more differentiation between the blood vessel and OC. The green channel, therefore, is selected for the extraction of the OC of the retinal image.

\subsubsection{Removal of Blood Vessels}

Blood vessels in the green channel were removed using a morphological closing procedure,

$$
I 2(I, B)=A \bullet B=E(D(I,-B),-B)
$$

where $\mathrm{I}$ is the green channel of the input image and $\mathrm{B}$ is an $8 \times 8$ symmetrical disc structuring element, to remove the blood vessels[13]. I2 is the smoothed, vessel free output image. Figure 2 shows the preprocessing operations on the input image. 


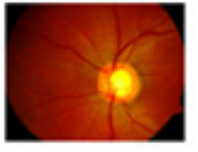

(a)

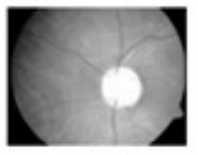

(b)

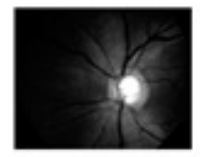

(d)

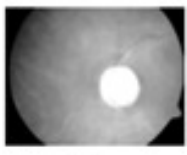

(c)

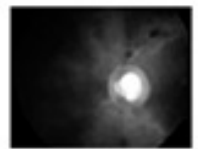

(e)

Figure 2. The preprocessing steps (a) Input Image (b) Red channel (c) Vessel free Image (d) Green chamel (e) Vessel free Image

\subsection{Feature Extraction}

Medical image segmentation is a difficult task due to the complexity of segmentation. Because of its simplicity and efficiency, threshold segmentation is wildly used in many fields. Assessment of $\mathrm{OD}$ and $\mathrm{OC}$ is important in discriminating between normal and pathological retinal images. The $\mathrm{OD}$ is a bright pattern of the fundus image. Recently, many studies on the use of fundus images in extracting OD and OC have been reported. Fuzzy C Means Clustering with thresholding is used in this work for the extraction of OD and OC.

\subsection{Fuzzy C Means Clustering with Thresholding}

The proposed method is a combination of fuzzy algorithm, C Means clustering and thresholding. Clustering involves the task of dividing data points into homogeneous classes or clusters so that items in the same class are as similar as possible and items in different classes are as dissimilar as possible. Clustering can also be thought of as a form of data compression, where a large number of samples are converted into a small number of representative prototypes or clusters. Different types of similarity measures may be used to identify classes depending on the data and the application, where the similarity measure controls the formation of the clusters. In the following new method intensity value is used as the similarity measure. Thresholding is one of the most powerful techniques for image segmentation, in which the pixels are partitioned depending on their intensity value.

\subsubsection{Fuzzy C-Means Clustering Algorithm}

Fuzzy C-Means (FCM) Clustering is a clustering technique and it employs fuzzy partitioning such that a data point can belong to all groups with different membership grades between 0 and 1 . It is an iterative algorithm. The aim of FCM is to find cluster centers (centroids) that minimize a dissimilarity function. Corresponding to each cluster center, this algorithm works by assigning membership to each data point on the basis of the difference between the cluster center and the data point. The more the data is near to the cluster center, the more is its membership towards the particular cluster center. It is obvious that the summation of membership of each data point should be equal to one.

$$
\begin{aligned}
& \sum_{i=1}^{c} u_{i j}=1, \forall j=1, \ldots, n \\
& \mathrm{~d}_{\mathrm{ik}}=\left[\sum_{\mathrm{j}=1}^{\mathrm{m}}\left[\mathrm{x}_{\mathrm{kj}}-\mathrm{v}_{\mathrm{j}}\right]^{2}\right]^{1 / 2},
\end{aligned}
$$


where $\mathrm{x}_{\mathrm{kj}}$ is data element, $\mathrm{d}_{\mathrm{ik}}$ is the distance matrix and $\mathrm{v}_{\mathrm{ij}}$ is the element of the cluster center vector.

The dissimilarity function which is used in FCM is given Equation (5)

$$
J\left(U, c_{1}, c_{2}, \ldots, c_{c}\right)=\sum_{i=1}^{c} J_{i}=\sum_{i=1}^{c} \sum_{j=1}^{n} u_{i j}^{m} d_{i j}^{2}
$$

uij is between 0 and 1 ;

$c i$ is the centroid of cluster $\mathrm{i}$;

dij is the Euclidian distance between ith centroid(ci) and jth data point;

$m \epsilon[1, \infty]$ is a weighting exponent.

To reach a minimum of dissimilarity function there are two conditions. These are given in Equation (6) and Equation (7)

$$
\begin{gathered}
c_{i}=\frac{\sum_{j=1}^{n} u_{i j}{ }^{m} x_{j}}{\sum_{j=1}^{n} u_{i j}{ }^{m}} \\
u_{i j}=\frac{1}{\sum_{k=1}^{c}\left(\frac{d_{i j}}{d_{k j}}\right)^{2 /(m-1)}}
\end{gathered}
$$

This algorithm determines the following steps [4].

Step1. Randomly initialize the membership matrix (U) that has constraints in Equation 7.

Step2. Calculate centroids $\left(\mathrm{C}_{\mathrm{i}}\right)$ by using Equation(6).

Step3. Compute dissimilarity between centroids and data points using equation (5). Stop if its improvement over previous iteration is below a threshold.

Step4. Compute a new U using Equation(7). Go to Step 2 [16][17].

By iteratively updating the cluster centers and the membership grades for each data point, FCM iteratively moves the cluster centers to the apt location within a data set. To accommodate the introduction of fuzzy partitioning, the membership matrix $(\mathrm{U})$ is randomly initialized according to Equation (7).

The Fuzzy Logic Toolbox command line function $f \mathrm{~cm}$ is used for generating clusters, and in this paper three clusters are generated from the vessel free enhanced image. The $f c m$ function iteratively moves the cluster centers to the right location within the data set. The outputs are 3 cluster centers $\mathrm{C} 1, \mathrm{C} 2$ and $\mathrm{C} 3$ and membership function matrix $\mathrm{M}$ with membership-grades, which is the intensity value of each pixel. 


\subsection{Thresholding}

Thresholding is the operation of converting a multilevel image into a binary image i.e., it assigns the value of 0 (background) or 1 (objects or foreground) to each pixel of an image based on a comparison with some threshold value $\mathrm{T}$ (intensity or colour value) [13][14][15]. By applying the threshold $\mathrm{T}$ on an image, the image is converted to a binary image. The following formula (8) [13] is used for the binary image extraction.

$$
\mathrm{I}_{\mathrm{T}}(\mathrm{x}, \mathrm{y})=\left\{\begin{array}{l}
1, \text { if } \mathrm{I}(\mathrm{x}, \mathrm{y})>\mathrm{T} \\
0, \text { if } \mathrm{I}(\mathrm{x}, \mathrm{y})<=\mathrm{T}
\end{array}\right.
$$

where $I$ is the input image, $\mathrm{T}$ is the threshold and $\mathrm{I}_{\mathrm{T}}$ is the binary image after thresholding.

\subsection{Extraction of Optic Disc}

The main feature of the OD is that it is having the highest intensity. Therefore the highest intensity is used as the threshold for the OD extraction. The threshold $\mathrm{T}$ is computed using the following method. From the generated clusters, first the cluster with maximum membership grade is found, and the corresponding grades are assigned with the same identification label. From the smoothed image, pixels with this gray level value are accessed, the average of the maximum and minimum intensity values are computed to obtain the threshold value $T_{1}$.

$$
\text { i.e., } \mathrm{T}_{1}=\frac{1}{2}[\operatorname{Max}(\text { data }(\text { value }))+\operatorname{Min}(\text { data }(\text { value }))]
$$

In the above equation, data represents the data points of the smoothed red channel image and label represents the cluster value with the highest membership grade. By applying the threshold $T_{1}$ on the smoothed image $I_{S}$ the image is converted to a binary image. The formula (9) is used for the binary image extraction.

Since the OD is of circular shape, the OD region selection process needs to be made specific to the circular region. So the largest connected component $R i$ whose shape is approximately circular is selected using the compactness measure

$$
\mathrm{C}(\mathrm{Ri})=\frac{\mathrm{P}(\mathrm{Ri})}{4 \pi \mathrm{A}(\mathrm{Ri})}
$$

where, $\mathrm{P}(\mathrm{Ri})$ is the perimeter of the region $R i$ and $\mathrm{A}(\mathrm{Ri})$ is the area of the region $R i$. The binary image with the compactness smaller than the pre-specified value, ( 5 in the present study) is considered as the optic disc approximation. Thus using the condition $\mathrm{C}<5$, extraction of round objects is done, eliminating those objects that do not meet the criteria. In some cases the extracted image contains small unwanted objects. The erosion operation is used to remove these objects.

The mean of the rows and columns form the centroid (Y1, X1) of the OD.

$$
\begin{aligned}
& \mathrm{Y} 1=\frac{1}{\mathrm{~m}} \sum_{\mathrm{i}=1}^{\mathrm{m}} \operatorname{row} 1 \mathrm{i} \\
& \mathrm{X} 1=\frac{1}{\mathrm{n}} \sum_{\mathrm{i}=1}^{\mathrm{n}} \operatorname{col} 1 \mathrm{i},
\end{aligned}
$$

where $m$ is the number of rows and $n$ is the number of columns. 
From the above coordinates of the optic disc the minimum coordinates (ymin1, xmin 1 ) is calculated. The distance between the centroid and (ymin1, xmin1) represents the radius of the disc.

$$
\mathrm{R}_{\mathrm{OD}}=\mathrm{Y} 1-\mathrm{ymin} 1,
$$

where $\mathrm{R}_{\mathrm{OD}}$ is the radius of the optic disc.

\subsection{Optic Disc Segmentation}

OD segmentation obtains a circular boundary approximation within a retinal image. A circle is plotted using the centroid $(\mathrm{Y} 1, \mathrm{X} 1)$ and radius $\mathrm{R}_{\mathrm{OD}}$, gives segmented $\mathrm{OD}$ on the colour fundus eye image. Figure 3 shows the segmented OD [18].

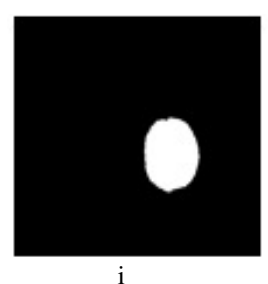

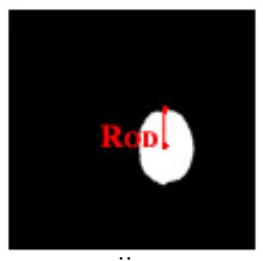

ii

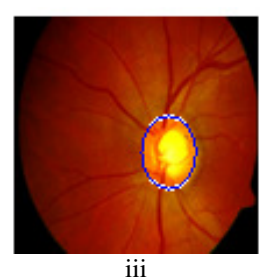

iii

Figure 3 i. Extracted optic disc ii. Centre and radius of extracted disc iii. Optic disc segmentation

\subsection{Extraction of Optic Cup}

The above mentioned FCM clustering with thresholding is applied on the smoothed green channel for the extraction of OC.

The following algorithm includes four steps [4].

Step1. Randomly initialize the membership matrix (U) that has constraints in Equation (6).

Step2. Calculate centroids (Ci) by using Equation (7).

Step3. Compute dissimilarity between centroids and data points using equation (5). Stop if its improvement over previous iteration is below a threshold.

Step4. Compute a new U using Equation (6). Go to Step 2[16][17].

The threshold values $T_{2}$ is calculated using the following equation.

$$
\mathrm{T}_{2}=\frac{1}{2}[\operatorname{Max}(\operatorname{data}(\text { value }))+\operatorname{Min}(\text { data }(\text { value }))]
$$

where data represents the data points of the vessel free green channel and label represents the cluster value with the highest membership grade.

Since the OC is the brightest portion in the green channel, thresholding with threshold $T_{2}$ in im2bw function helps to extract OC. This function returns the binary image forming the object OC. 
The average of the rows and columns forms the centroid (Y2, X2) of the OC.

$$
\begin{aligned}
& \mathrm{Y} 2=\frac{1}{\mathrm{~m} 2} \sum_{\mathrm{i}=1}^{\mathrm{m} 2} \text { row } 2 \mathrm{i} \\
& \mathrm{X} 2=\frac{1}{\mathrm{n} 2} \sum_{\mathrm{i}=1}^{\mathrm{n} 2} \operatorname{col} 2 \mathrm{i}
\end{aligned}
$$

where $m 2$ is the number of rows and $n 2$ is the number of columns.

From the above coordinates of the optic cup the minimum coordinates (ymin2, xmin2) is calculated. The Euclidian distance between the centroid and (ymin1, xmin1) returns the radius of the cup.

$$
\mathrm{R}_{\mathrm{OC}}=\mathrm{Y} 2-\mathrm{ymin} 2,
$$

where $\mathrm{R}_{\mathrm{OC}}$ is the radius of the cup.

\subsection{Optic Cup Segmentation}

OC within OD usually appears in circular shape. Therefore the OC segmentation is a circular boundary approximation. Using the centroid $(\mathrm{Y} 2, \mathrm{X} 2)$ and radius $\mathrm{R}_{\mathrm{OC}}$ a circle is drawn onto the current axes of the input image which would give the segmented OC on the colour fundus eye image.

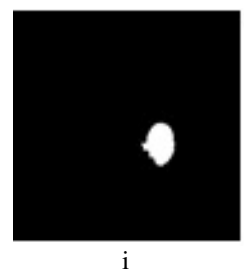

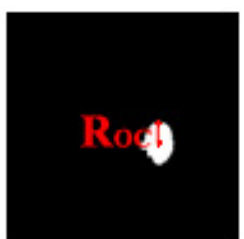

ii

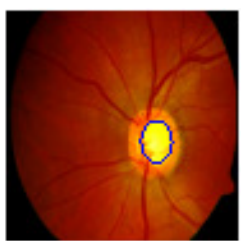

iii

Figure 4 i. Extracted Optic cup ii. Localization of centre and radius of optic cup iii. Optic cup segmentation

\subsection{Computation of CDR}

The manual method uses the ratio of the vertical diameter of OC and OD for the computation of $\mathrm{CDR}$. From the segmented OD the minimum row coordinate $\mathrm{y}_{\min l}$ and maximum row coordinate $\mathrm{y}_{\max 1}$ are calculated. The Euclidian distance between these coordinates is the vertical diameter of the OD, ODvdiam .

$$
\mathrm{OD} v \text { diam }=y \max 1-y \min 1
$$

Similarly from the segmented $\mathrm{OC}$ the minimum row coordinate $\mathrm{y}_{\min 2}$ and maximum row coordinate $\mathrm{y}_{\max 2}$ are calculated. The Euclidian distance between these coordinates is the vertical diameter of the OC, OCvdiam.

$$
\mathrm{OC} \text { diam }=y \max 2-y \min 2
$$

The CDR is calculated using the following formula

$$
\mathrm{CDR}=\mathrm{OC} v \text { diam } / \mathrm{OD} v \text { diam }
$$


The following figure shows the OD vertical diameter ODvdiam and OC vertical diameter OCvdiam of the input image.

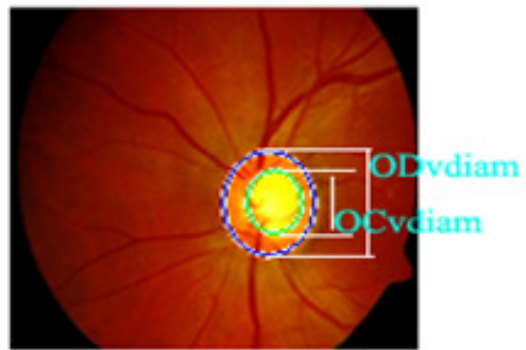

Figure5 Optic Disc vertical diameter ODvdiam and Optic Cup vertical diameter OCvdiam

\section{RESULTS AND DISCUSSION}

The automatic detection and evaluation of OD and OC is required for automatic diagnosis using retinal images. This study thus brings to light simple but efficient methods for the extraction and segmentation of OD and OC in retinal images. The CDR values are also automatically calculated. The new method is evaluated on the basis of the ground truth data, where, vertical CDR values are obtained from an expert ophthalmologist. Four hundred and fifty four color retinal images, including thirty normal and four hundred and thirty three pathological images, are used in this test. The performance evaluation is done by making use of the scatter plot analysis.

\subsection{Image Data Sets}

\subsubsection{The DIARETDB0 and DIARETDB1 Databases}

The DIARETDB0 and DIARETDB1 Database images were captured using an FOV of $50^{\circ}$ and the size of each image is $1500 \times 1152 \times 3$. Out of the 130 images of the DIARETDB0 database, 20 have normal architecture and 110 have various types of pathology. Out of the 89 images of the DIARETDB1 database, 5 have normal architecture and 84 have various types of pathology.

\subsubsection{DRION Database}

It has 110 retinal images with each image having the resolution of $600 \times 400$ pixels and the optic disc annotated by two experts with 36 landmarks. The mean age of the patients was 53.0 years (standard Deviation 13.05), with $46.2 \%$ male and $53.8 \%$ female and all of them were Caucasian ethnicity $23.1 \%$ patients had chronic simple glaucoma and $76.9 \%$ eye hypertension. The images were acquired with a colour analogical fundus camera, approximately centered on the $\mathrm{ONH}$ and they were stored in slide format. In order to have the images in digital format, they were digitized using a HP-PhotoSmart-S20 high-resolution scanner, RGB format, resolution 600x400 and 8 bits/pixel.

\subsubsection{Images from the ophthalmologist}

125 images from Giridhar Eye Institute, Kochi was also used in this paper. All the images were obtained using Carlzeiss fundus camera. In total 5 are normal images and remaining 120 are diseased and the size of each image is $576 \times 768 \times 3$. 


\subsection{Implementation}

The new algorithm was applied on 454 images obtained from the above mentioned databases and ophthalmologists. Seven of the input images from each data set, along with their OD and OD segmentation on the input image, is shown in fig.6 (a) and fig.6 (b) respectively.

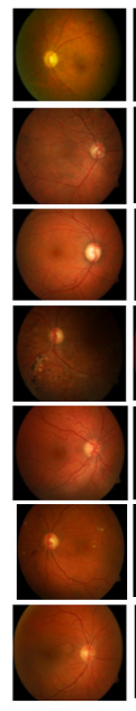

(a)

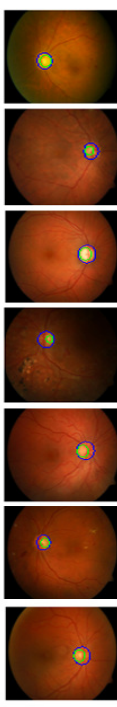

(b)

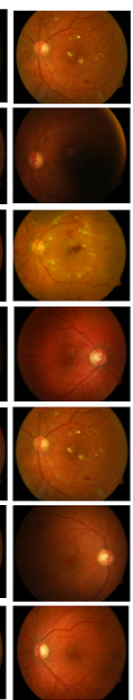

(c)

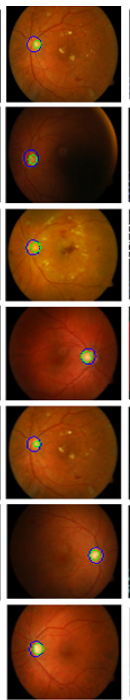

(d)

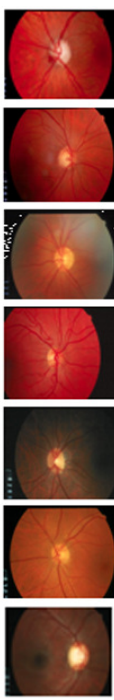

(e)

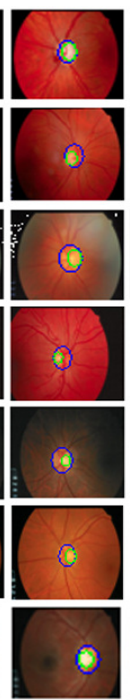

(f)

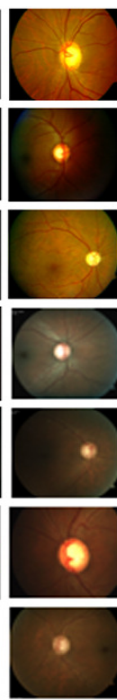

(g)
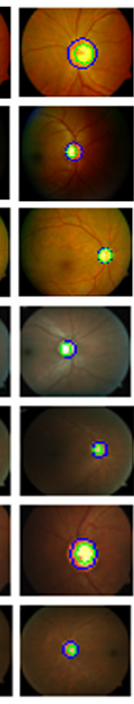

(h)

Figure 6 Input Image and OD \& OC segmentation using new method on images from (a) and(b) DIARATDBO (e) and(f) DRION (c) and(d)

\subsection{Performance Evaluation}

The performance evaluation is done using the following parameters.

\subsubsection{Success rate}

The decision for successful segmentation or failed segmentation is based on human eye observation. Table 1 shows the success rate of OD and OC segmentation using 454 images.

Table1. Success Rate Table

\begin{tabular}{|l|c|c|c|c|}
\hline \multicolumn{1}{|c|}{ Database } & Normal & Pathological & Total & $\begin{array}{c}\text { Success Rate } \\
(\%)\end{array}$ \\
\hline Drion & 0 & 110 & 110 & 94.5 \\
\hline Diaretdb0 & 20 & 110 & 130 & 95.4 \\
\hline Diaretdb1 & 5 & 84 & 89 & 93.3 \\
\hline Ophthalmologist & 5 & 129 & 125 & 97.3 \\
\hline Total & 30 & 433 & 454 & $\begin{array}{c}94.26 \\
\text { (Average) }\end{array}$ \\
\hline
\end{tabular}

\subsubsection{Accuracy}

The accuracy of the technique was evaluated quantitatively by comparing the obtained vertical CDR values with ophthalmologists' ground-truth vertical CDR values. Fifteen examples of 
detailed results of performance measurement using FCM clustering combined with thresholding are displayed in Table II using fifteen test images of DRION database and fifteen test images from the ophthalmologist.

Table II CDR Comparison Table shows the comparison of clinical CDR values with CDR values obtained using the new method.

\begin{tabular}{|l|l|l|l|l|l|l|}
\hline \multicolumn{1}{|c|}{ Images } & $\begin{array}{c}\text { Clinical } \\
\text { CDR } \\
\mathbf{( 1 )}\end{array}$ & $\begin{array}{l}\text { Obtained } \\
\text { CDR } \\
(2)\end{array}$ & $\begin{array}{l}\text { Difference } \\
\mathbf{( 1 )}-(\mathbf{2})\end{array}$ & $\begin{array}{l}\text { Clinical } \\
\text { CDR } \\
\text { DRION } \\
\text { Database } \\
(\mathbf{3})\end{array}$ & $\begin{array}{l}\text { obtained } \\
\text { CDR }\end{array}$ & $\begin{array}{l}\text { Difference } \\
\mathbf{( 3 )} \text {-(4) }\end{array}$ \\
\hline Image 1 & 0.5000 & 0.6082 & 0.1082 & 0.3333 & 0.4000 & 0.0667 \\
\hline Image 2 & 0.5714 & 0.6231 & 0.0517 & 0.5734 & 0.5310 & 0.0424 \\
\hline Image 3 & 0.6666 & 0.5505 & 0.1161 & 0.6666 & 0.6080 & 0.0586 \\
\hline Image 4 & 0.8517 & 0.7871 & 0.0646 & 0.7000 & 0.7543 & 0.0543 \\
\hline Image 5 & 0.7142 & 0.6412 & 0.073 & 0.6578 & 0.5816 & 0.0762 \\
\hline Image 6 & 0.4864 & 0.4173 & 0.0691 & 0.5625 & 0.5045 & 0.0580 \\
\hline Image 7 & 0.7060 & 0.6275 & 0.0785 & 0.4062 & 0.4995 & 0.0933 \\
\hline Image 8 & 0.9000 & 0.8367 & 0.0633 & 0.5000 & 0.6020 & 0.1020 \\
\hline Image 9 & 0.6801 & 0.7287 & 0.0486 & 0.6097 & 0.6696 & 0.0626 \\
\hline Image 10 & 0.9026 & 0.8206 & 0.0820 & 0.6410 & 0.6610 & 0.0200 \\
\hline Image 11 & 0.4631 & 0.4012 & 0.0619 & 0.5162 & 0.4011 & 0.1151 \\
\hline Image 12 & 0.6267 & 0.5151 & 0.1116 & 0.5010 & 0.5362 & 0.0352 \\
\hline Image 13 & 0.5147 & 0.4736 & 0.0411 & 0.6097 & 0.5215 & 0.0882 \\
\hline Image 14 & 0.7318 & 0.7180 & 0.0138 & 0.7408 & 0.6943 & 0.0465 \\
\hline Image 15 & 0.4265 & 0.3162 & 0.1103 & 0.6981 & 0.6208 & 0.0773 \\
\hline Mean Differen & & & 0.07292 & Mean Difference & 0.06643 \\
\hline
\end{tabular}

From the table it is shown that the mean differences between the clinical CDR values and obtained CDR values are very low.

\subsubsection{Scatter plot Analysis}

The statistical analysis is done using the scatter plot diagram. The clinical CDR vales and the obtained CDR values of the above mentioned data set are analysed using the scatter plot analysis. From the diagram it is shown that there exists highly positive linear relationship between both CDR values. Figure7 depicts the comparison between the clinical CDR and obtained CDR values. 


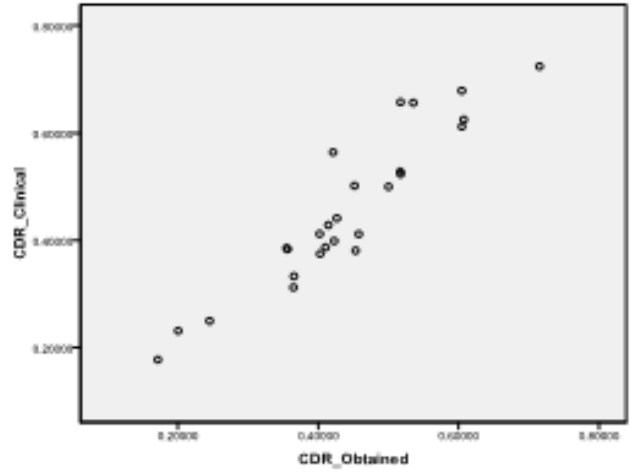

(a)

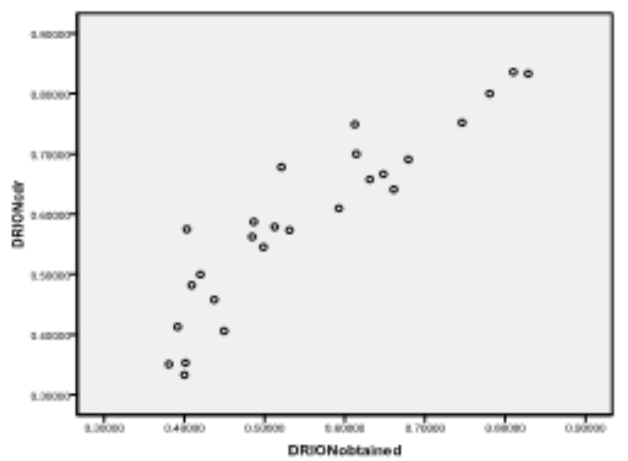

(c)

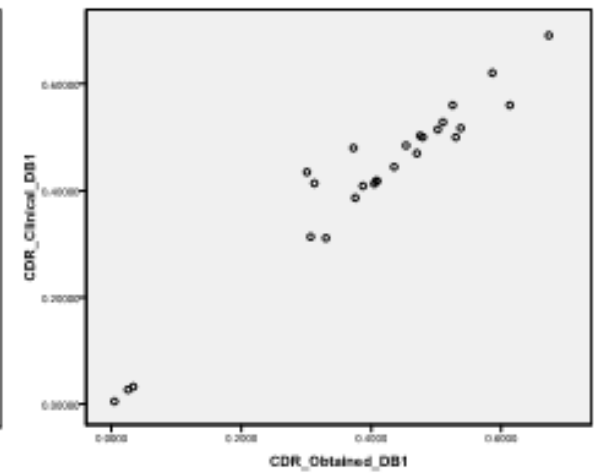

(b)

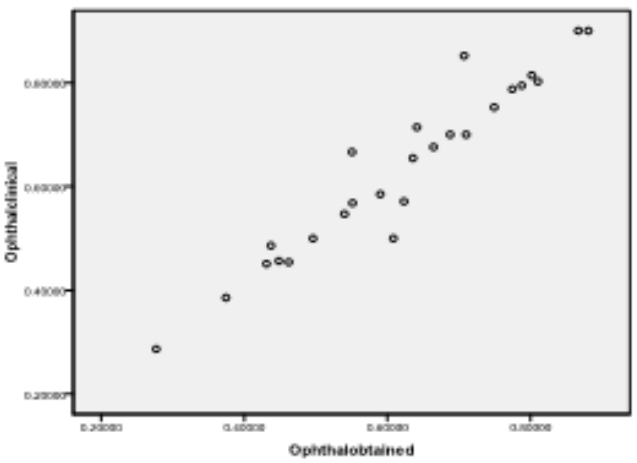

(d)

Figure7. Scatter Plot Diagram (a) Diaretdb0 (b) Diaretdb1 (c) Drion (d) Ophthalmologist

\section{CONCLUSION}

This paper presents a new fuzzy based approach for OD and OC extraction and segmentation together with CDR computation. The results presented in this paper show that the new methodology offers a reliable and robust solution for CDR computation. The scatter plot analysis results a high positive correlation between the clinical CDR and the obtained CDR. This automated method is very useful for the automatic screening of retinal images. However the present method has the following limitations. It is assumed that the OD and OC are brighter than the surrounding pixels and therefore cannot handle retinal images with a relatively dark OD. Hence advanced extraction methods are required for future studies and research.

\section{REFERENCES}

[1] Juan Xu, Hiroshi Ishikawa, Gadi Wollstein, Richard A. Bilonick, Kyung R. Sung,Larry Kagemann, Kelly A. Townsend, and Joel S. Schuman, "Automated Assessment of the Optic Nerve Head on Stereo Disc Photographs”, Invest Ophthalmol Vis Sci. Jun 2008; 49(6), pp. 2512-2517, 2008.

[2] Thitiporn Chanwimaluang and Guoliang Fan, "An efficient algorithm for extraction of anatomical structures in retinal images", Proc. of International Conference on Image Processing, Vol. 1, pp. 1093-1096,2003

[3] Chisako Muramatsu, Toshiaki Nakagawab, Akira Sawadac, Yuji Hatanakad, Takeshi Haraa, Tetsuya Yamamotoc, Hiroshi Fujitaa "Determination of cup and disc ratio of optical nerve head for diagnosis 
of glaucoma on stereo retinal fundus image pairs", Medical Imaging 2009: Computer-Aided Diagnosis, Proc. of SPIE ,Vol. 7260, pp.72603L-1 - 72603L-8, 2009

[4] Huiqi Li, Opas Chutatape "Boundary detection of optic disc by a modified ASM method", The Journal of the Pattern Recognition Society, Vol. 36, pp. 2093-2104,2003

[5] Huajun Ying, Ming Zhang and Jyh-Charn Liu, "Fractal-Based Automatic Localization and Segmentation of Optic Disc in Retinal Images", 28th Annual International Conference of the IEEE Engineering In Medicine and Biology Society (EMBS), 2007

[6] S. Sekhar, "Automated Localization Of Optic Disk And Images", Proc. 16th European Signal Processing Conference (EUSIPCO 2008), Lausanne, Switzerland, August 25-29, 2008.

[7] Hussain, A.R., "Optic nerve head segmentation using genetic active Contours", International Conference on Computer and Communication Engineering, 2008,ICCCE 2008, 13-15 May 2008, pp.783 - 787, Univ. Teknikal Malaysia, Melaka, May 2008.

[8] Zhuo Zhang ,Jiang Liu, Cherian, N.S., Ying Sun, Joo Hwee Lim, Wing Kee Wong, Ngan Meng Tan ,Shijian Lu , Huiqi Li , Tien Ying Wong, "Convex hull based neuro-retinal optic cup ellipse optimization in glaucoma diagnosis", Engineering in Medicine and Biology Society, 2009. EMBC2009. Annual International Conference of the IEEE, Inst. for Infocomm Res., A*STAR, Singapore, 3-6 Sept. 2009, pp. 1441 - 1444, 2009.

[9] Wong, D.W.K. ; Jiang Liu ; Joo Hwee Lim ; Ngan Meng Tan ; Zhuo Zhang ; Huiqi Li ; Shijian Lu ; Tien Yin Wong, 'Method of detecting kink-bearing vessels in a retinal fundus image(CDR)',The 5th IEEE Conference on Industrial Electronics and Applications (ICIEA), pp. 1690 - 1694, June 2010

[10] Joshi, G.D., Sivaswamy, J., Karan, K., Prashanth, R., Krishnadas, S.R., "Vessel Bend-Based Cup Segmentation in Retinal Images", 20th International Conference on Pattern Recognition (ICPR), 2010, CVIT, IIIT Hyderabad, Hyderabad, India, 23-26 Aug. 2010, pp. 2536 - 2539, 2010.

[11] Shijian Lu and Joo Hwee Lim, "Automatic optic disc detection through background estimation" Proceedings of 2010 IEEE 17th International Conference on Image Processing, Hong Kong, September 26-29, 2010.

[12] Angel Suero, Diego Marin, Manuel E. Gegundez-Arias, and Jose M. Bravo, "Locating the Optic Disc in Retinal Images Using Morphological Techniques", IWBBIO 2013 Proceedings, Granada, 18-20 March, 2013, pp.593-600, 2013.

[13] Rafael C Gonzalez, Richard E Woods, Steven L Eddins, Digital Image Processing, Prentice Hall Publications, 2008.

[14] N. M. Salem and A. K. Nandi, "Novel and adaptive contribution of the red channel in pre-processing of colour fundus images," in Journal of the Franklin Institute, 2007, p. 243256, 2007.

[15] Rafael C Gonzalez, Richard E Woods, Steven L Eddins., Digital Image Processing Using Matlab, Prentice Hall Publications, 2008.

[16] HeikoTimm, Christian Borgelt, and Rudolf KruseFuzzy, "Cluster Analysis with Cluster Repulsion", CiteSeerx.

[17] Yinghua Lu, Tinghuai Ma, Changhong Yin, Xiaoyu Xie, Wei Tian,ShuiMing Zhong, "Implementation of the Fuzzy C-Means Clustering Algorithm in Meteorological Data", International Journal of Database Theory and Application, Vol.6, No.6, pp.1-18, 2013

[18] Thresiamma Devasia, Poulose Jacob, Tessamma Thomas, "Automatic Optic Disc Boundary Extraction from Color Fundus Images", International Journal of Advanced Computer Science and Applications (IJACSA), Vol. 5, No. 7, 2014,pp.17-24

[19] Yong Yang, Shuying Huang, " Image segmentation by fuzzy c-means Clustering algorithm with a novel Penalty term”, Computing and Informatics, Vol. 26, pp. 17-31, 2007. 


\section{AUTHORS}

Thresiamma Devasia was graduated with Bachelor of Mathematics (BSc.Maths) from Mahatma University, Kerala, India in 1991, and finished her Master of Computer Applications (MCA) and M.Phil Computer Science from Alagappa University Tamilnadu, India in 1995 and 2010, respectively.

Currently, she is the Head and Associate professor, Department of Computer Science at Assumption College Changanacherry, Kerala, India and working toward her Ph.D. at Cochin University of Science And Technology on glaucoma detection using image

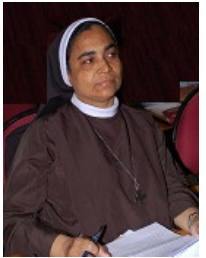
processing.

She completed UGC sponsored minor research project based on image processing. She was a member of IEEE. Her interest areas include image processing and medical imaging.

Dr. K.Poulose Jacob, Professor of Computer Science at Cochin University of Science and Technology since 1994, is currently Pro Vice Chancellor of Cochin University of Science \& Technology. He has presented research papers in several International Conferences in Europe, USA, UK, Australia and other countries. He has served as a Member of the Standing Committee of the UGC on Computer Education \& Development. He is the Zonal Coordinator of the DOEACC Society under the Ministry of Information Technology, Government of India. He serves as a member of the AICTE

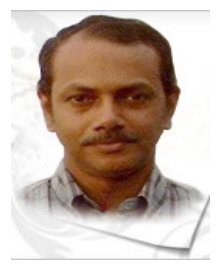
expert panel for accreditation and approval. He has been a member of several academic bodies of different Universities and Institutes. He is on the editorial board of two international journals in Computer Science. Dr. K.Poulose Jacob is a Professional member of the ACM (Association for Computing Machinery) and a Life Member of the Computer Society of India.

Dr.Tessamma Thomas received her M.Tech. and Ph.D from Cochin University of Science and Technology, Cochin-22, India. At present she is working as Professor in the Department of Electronics, Cochin University of Science and Technology. She has to her credit more than 100 research papers, in various research fields, published in International and National journals and conferences. Her areas of interest include digital signal / image processing, bio medical image processing, super resolution, content based image retrieval, genomic signal processing, etc.

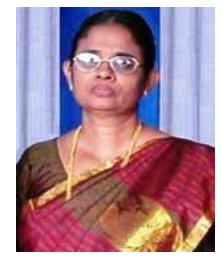

